\title{
TATE PROPERTIES, POLYNOMIAL-COUNT VARIETIES, AND MONODROMY OF HYPERPLANE ARRANGEMENTS
}

\author{
ALEXANDRU DIMCA
}

\begin{abstract}
The order of the Milnor fiber monodromy operator of a central hyperplane arrangement is shown to be combinatorially determined. In particular, a necessary and sufficient condition for the triviality of this monodromy operator is given.

It is known that the complement of a complex hyperplane arrangement is cohomologically Tate and, if the arrangement is defined over $\mathbb{Q}$, has polynomial count. We show that these properties hold for the corresponding Milnor fibers if the monodromy is trivial.

We construct a hyperplane arrangement defined over $\mathbb{Q}$, whose Milnor fiber has a nontrivial monodromy operator, is cohomologically Tate, and has no polynomial count. Such examples are shown not to exist in low dimensions.
\end{abstract}

\section{$\S 1$. Introduction}

Let $\mathcal{A}$ be a central arrangement of $d$ hyperplanes in $\mathbb{C}^{n+1}$, with $d \geq 2$ and $n \geq 1$, given by a reduced equation $Q(x)=0$. Consider the corresponding global Milnor fiber $F$ defined by $Q(x)-1=0$ in $\mathbb{C}^{n+1}$ with monodromy action $h: F \rightarrow F, h(x)=\exp (2 \pi i / d) \cdot x$.

A general investigation line is to check whether certain properties of the associated projective hyperplane arrangement complements $M(\mathcal{A})$ in $\mathbb{P}^{n}$ extend to the Milnor fiber $F$. For instance, note the following.

(i) The cohomology ring $H^{*}(M(\mathcal{A}), \mathbb{Z})$ is determined by the combinatorics (see [20]), but the same question for the Betti numbers of the Milnor fiber $F$ is widely open (see, e.g., [16]).

(ii) $H^{*}(M(\mathcal{A}), \mathbb{Z})$ is torsion-free (see $\left.[20]\right)$, and there is the open question about the torsion-freeness of $H^{*}(F, \mathbb{Z})$ (see [2]).

Received May 5, 2011. Accepted August 23, 2011.

2010 Mathematics Subject Classification. Primary 32S22, 32S35; Secondary 32S25, $32 \mathrm{~S} 55$.

The author's work was partially supported by Agence Nationale de la Recherche grant ANR-08-BLAN-0317-02 (SEDIGA).

(C) 2012 by The Editorial Board of the Nagoya Mathematical Journal 
(iii) The complement $M(\mathcal{A})$ is formal in the sense of Sullivan ([28], [4]), and it was recently shown that the Milnor fiber $F$ may be not even 1-formal (see [30]).

We say that a complex variety $Y$ is cohomologically Tate if for any cohomology group $H^{m}(Y, \mathbb{C})$, one has the following vanishing of mixed Hodge numbers: $h^{p, q}\left(H^{m}(F, \mathbb{C})\right)=0$ for $p \neq q$. The fact that the hyperplane arrangement complements $M(\mathcal{A})$ are cohomologically Tate has been known for a long time: any cohomology group $H^{m}(M(\mathcal{A}), \mathbb{Q})$ is a pure Hodge structure of type $(m, m)$ (see [14], [12], [25]).

When the monodromy action $h^{*}$ is trivial on all the cohomology groups $H^{*}(F, \mathbb{C})$, it follows that we have an equality $H^{m}(F, \mathbb{Q})=H^{m}(M(\mathcal{A}), \mathbb{Q})$ for any $0 \leq m \leq n$, and hence in this case $F$ is cohomologically Tate. One may ask whether this is the only possibility for a hyperplane arrangement Milnor fiber $F$ to be cohomologically Tate. The claim that $F$ cohomologically Tate implies $h^{*}$ trivial is shown to be true in the case $n=1$ (obvious, using the mixed Hodge structure (MHS) on the Milnor fiber of an isolated homogeneous hypersurface singularity, given by Steenbrink [27] and recalled in [5, pp. 243-244]) and $n=2$, that is, for plane arrangements, and negative in general. We do not know whether there is a similar result to Theorem 1.1 for $2<n<7$, and this explains why we go to dimension 7 to construct our example.

To state our results in this direction, we need some preliminaries.

In studying the cohomology $H^{*}(F, \mathbb{Q})$ of the Milnor fiber, the monodromy action $h^{*}: H^{*}(F, \mathbb{Q}) \rightarrow H^{*}(F, \mathbb{Q})$, or the number of points in $\left|F\left(\mathbb{F}_{p}\right)\right|$, we can, without any loss of generality, suppose that the arrangement $\mathcal{A}$ is essential; that is, $\bigcap_{H \in \mathcal{A}} H=0$. This is the same as supposing that the polynomials $Q$ involve in an essential way all the variables $x_{0}, \ldots, x_{n}$; that is, one cannot choose the coordinates $x$ on $\mathbb{C}^{n+1}$ such that $Q\left(x_{0}, \ldots, x_{n}\right)=R\left(x_{0}, \ldots, x_{u}\right)$ for some $0 \leq u<n$ and a homogeneous polynomial $R \in \mathbb{C}\left[x_{0}, \ldots, x_{u}\right]$.

The properties of the monodromy $h^{*}: H^{*}(F, \mathbb{Q}) \rightarrow H^{*}(F, \mathbb{Q})$ are rather mysterious, and many things that we know in general are related to the spectrum

$$
\operatorname{Sp}(\mathcal{A})=\sum_{\alpha \in \mathbb{Q}} m_{\alpha} t^{\alpha}
$$

with $m_{\alpha}=\sum_{j}(-1)^{j-n} \operatorname{dim} G r_{F}^{p} \tilde{H}^{j}(F, \mathbb{C})_{\beta}$, where $p=[n+1-\alpha]$ and $\beta=$ $\exp (-2 \pi i \alpha)$, which is combinatorially determined (see [1]). Surprisingly, 
note that for most arrangements the situation is rather simple; namely, $h^{m}: H^{m}(F, \mathbb{Q}) \rightarrow H^{m}(F, \mathbb{Q})$ is trivial (i.e., the identity) for $0 \leq m<n$, and $\operatorname{dim} H^{n}(F, \mathbb{C})_{\beta}=|\chi(M(\mathcal{A}))|$, for any $\beta \in \mu_{d}=\left\{z \in \mathbb{C} \mid z^{d}=1\right\}$ with $\beta \neq 1$ (see, e.g., [3], [15], [6, Propositions 2.5.4, 6.4.6, Example 6.4.14, Theorem 6.4.18]).

TheOREM 1.1. Let $\mathcal{A}$ be an essential central arrangement of $d$ planes in $\mathbb{C}^{3}$. The following conditions are equivalent.

(i) The mixed Hodge numbers $h^{p, q}\left(H^{2}(F, \mathbb{C})\right)$ vanish for $p \neq q$.

(ii) The arrangement $\mathcal{A}$ is reducible.

(iii) The monodromy action $h^{*}$ is trivial on all the cohomology groups $H^{*}(F, \mathbb{C})$.

(iv) The spectral numbers $m_{\alpha}$ in $\operatorname{Sp}(\mathcal{A})$ vanish for all $\alpha \in(0,1)$.

Geometrically, property (ii) means the following: in the projective line arrangement $\mathcal{A}^{\prime}$ associated to $\mathcal{A},(d-1)$ lines meet in one point, say, $A$, and the remaining line $L_{d}$ does not contain $A$.

In terms of coordinates, this means that one may choose the coordinates $(x: y: z)$ on $\mathbb{P}^{2}$ such that $A=(0: 0: 1)$ and $L_{d}: z=0$. With this choice, one has $Q(x, y, z)=Q_{1}(x, y) z$, where $Q_{1}$ is a degree $(d-1)$ reduced homogeneous polynomial in $x, y$. This property is exactly the definition of a reducible arrangement when $n=2$.

To discuss the higher-dimensional case, we need the following precise characterization of arrangements with a trivial monodromy.

ThEOREM 1.2. For an essential central arrangement $\mathcal{A}$, the following conditions are equivalent.

(i) The monodromy action $h^{*}$ is trivial on all the cohomology groups $H^{*}(F, \mathbb{C})$.

(ii) The arrangement $\mathcal{A}$ is reducible and satisfies the following: if $\mathcal{A}=$ $\mathcal{A}_{1} \times \cdots \times \mathcal{A}_{q}$ is the decomposition of $\mathcal{A}$ as a product of irreducible arrangements, and if $d_{j}=\left|\mathcal{A}_{j}\right|$ denotes the number of hyperplanes in $\mathcal{A}_{j}$, then $\operatorname{GCD}\left(d_{1}, \ldots, d_{q}\right)=1$.

Moreover, if $\mathcal{A}$ is defined over $\mathbb{Q}$ (i.e., each hyperplane in $\mathcal{A}$ is defined over $\mathbb{Q})$, then all the irreducible arrangements $\mathcal{A}_{j}$ are also defined over $\mathbb{Q}$.

We show below (see Lemma 2.1) that in the (unique) decomposition $\mathcal{A}=$ $\mathcal{A}_{1} \times \cdots \times \mathcal{A}_{q}$ of $\mathcal{A}$ as a product of irreducible arrangements $\mathcal{A}_{j}$, the integers $q, d_{1}, \ldots, d_{q}$ are determined by the combinatorics, that is, by the intersection lattice $L(\mathcal{A})$ (see [21]). 
We recall that a central arrangement $\mathcal{A}$ as above is reducible if one can choose the coordinates $x$ on $\mathbb{C}^{n+1}$ such that $Q\left(x_{0}, \ldots, x_{n}\right)=R_{1}\left(x_{0}, \ldots, x_{u}\right) \times$ $R_{2}\left(x_{u+1}, \ldots, x_{n}\right)$ for some $0 \leq u<n$ and homogeneous nonconstant polynomials $R_{1}$ and $R_{2}$. We then write $\mathcal{A}=\mathcal{A}_{1} \times \mathcal{A}_{2}$, with $\mathcal{A}_{j}: R_{j}=0$.

It is known that an essential arrangement $\mathcal{A}$ is reducible if and only if $\chi(M(\mathcal{A}))=0($ see $[24])$. On the other hand, a trivial monodromy action $h^{*}$ implies that $\chi(M(\mathcal{A}))=0$, as a simple consequence of the general formula

$$
\sum_{j}(-1)^{j} \operatorname{dim} H^{j}(F, \mathbb{C})_{\beta}=\chi(M(\mathcal{A}))
$$

for any $\beta \in \mu_{d}$ (see $[6$, Propositions 2.5.4, 6.4.6]; see also $[1,(1.4 .2)]$ ).

REMARK 1.3. All compact Kähler manifolds are formal spaces (see [4]). When the monodromy $h^{*}$ is trivial, the corresponding Milnor fiber is clearly a formal space in Sullivan's sense (see [28], [9]). Hence, Theorem 1.2 yields a wealth of new examples of formal spaces in the class of smooth affine varieties. Note also that there are examples of nonformal smooth affine surfaces, related either to Milnor fibers of central plane arrangements (see [30]), or to isolated weighted homogeneous singularities (see [10]).

Theorem 1.2 is an obvious consequence of the following Thom-Sebastianitype result, where the sum of polynomials in disjoint sets of variables is replaced by their product. This result plays also a key role in the construction of our examples below.

Theorem 1.4. Let $\mathcal{A}=\mathcal{A}_{1} \times \cdots \times \mathcal{A}_{q}$ be the decomposition of the central essential arrangement $\mathcal{A}$ as a product of irreducible arrangements, let $d_{j}=$ $\left|\mathcal{A}_{j}\right|$ denote the number of hyperplanes in $\mathcal{A}_{j}$, and let $d_{0}=\operatorname{GCD}\left(d_{1}, \ldots, d_{q}\right)$. Then the following hold.

(i) There is a natural identification of graded MHS defined over $\mathbb{R}$,

$$
H^{*}(F, \mathbb{C})=H^{*}(\mathbb{T}, \mathbb{C}) \otimes\left(\bigoplus_{\beta \in \mu_{d_{0}}}\left(H^{*}\left(F_{1}, \mathbb{C}\right)_{\beta} \otimes \cdots \otimes H^{*}\left(F_{q}, \mathbb{C}\right)_{\beta}\right)\right)
$$

More precisely, for any $\beta \in \mu_{d_{0}}$, there is an identification

$$
H^{*}(F, \mathbb{C})_{\beta}=H^{*}(\mathbb{T}, \mathbb{C}) \otimes H^{*}\left(F_{1}, \mathbb{C}\right)_{\beta} \otimes \cdots \otimes H^{*}\left(F_{q}, \mathbb{C}\right)_{\beta}
$$

(ii) The monodromy operator $h^{*}: H^{*}(F, \mathbb{C}) \rightarrow H^{*}(F, \mathbb{C})$ has order $d_{0}$, which is determined by the intersection lattice $L(\mathcal{A})$. 
Since each $\mathcal{A}_{j}$ is irreducible, it follows from (1.2) that each $H^{*}\left(F_{j}\right)_{\beta}$ which occurs in Theorem 1.4 is nonzero.

REMARK 1.5. There are a number of papers dealing with ThomSebastiani-type results for the product of two polynomials $f$ and $g$ (or, more generally, for $h(f, g)$, with $h$ a function of two variables) (see, e.g., [18], [23], [17], [29]). The decomposition in our Theorem 1.4(i) appears in Tapp [29]. However, in all of these papers there is no reference to the MHS involved, and the methods used, being purely topological, do not allow us in a direct way to derive such conclusions. Since these MHS play a central role in the remainder of our paper, we give below a proof of this decomposition rather different from that in [29], carefully handling the corresponding MHS.

We have noticed already that $F$ is a cohomologically Tate variety when the monodromy action $h^{*}$ is trivial. We give below an example showing that the converse claim is false in general (see Example 4.3). Example 4.3 is also interesting since it shows that the part $H^{<\operatorname{top}}(F, \mathbb{C})_{\neq 1}$ of the Milnor fiber cohomology can be rather big, unlike all the previously known examples.

COROllary 1.6. Consider the central rational hyperplane arrangement $\mathcal{A}_{u, v}$ in $\mathbb{C}^{n+1}$ defined in Example 4.3, for any $u, v \in \mathbb{Z}_{>0}$. Then $n=3 u+$ $5 v-1$, the only eigenvalues of the monodromy operator $h^{*}$ are \pm 1 , and $\operatorname{dim} H^{*}\left(F_{u, v}, \mathbb{C}\right)_{-1}=2^{u+v-1}$. More precisely, the nontrivial $(-1)$-eigenspaces are exactly $H^{2 u+4 v+j}\left(F_{u, v}, \mathbb{C}\right)_{-1}$ for $0 \leq j \leq u+v-1$ and

$$
\operatorname{dim} H^{2 u+4 v+j}\left(F_{u, v}, \mathbb{C}\right)_{-1}=\left(\begin{array}{c}
u+v-1 \\
j
\end{array}\right) .
$$

The smallest $n$ for which our construction yields a counterexample is $n=7$; the Milnor fiber of the corresponding hyperplane arrangement $\mathcal{A}_{1,1}$ is cohomologically Tate, but $h^{*}$ is not trivial.

Recall that the (compactly supported) Hodge-Deligne polynomial (or Epolynomial) associated to a complex variety $Y$ is given by

$$
H D_{X}(x, y)=\sum_{u, v}\left(\sum_{j}(-1)^{j} h^{u, v}\left(H_{c}^{j}(Y, \mathbb{C})\right)\right) x^{u} y^{v} .
$$

Assume that $Y$ is in fact defined over $\mathbb{Q}$. We say that $Y$ has polynomial count with count polynomial $P_{Y}$ if there is a polynomial $P_{Y} \in \mathbb{Z}[t]$, such that for all but finitely many primes $p$, for any finite field $\mathbb{F}_{q}$ with $q=p^{s}$ and $s \in \mathbb{N}^{*}$, the number of points of $Y$ over $\mathbb{F}_{q}$ is precisely $P_{Y}(q)$. As an 
example, if the hyperplane arrangement $\mathcal{A}$ is defined over $\mathbb{Q}$, then $M(\mathcal{A})$ has polynomial count (see, e.g., [7, Section (5.3)], [26, Theorem 5.15]). The above rationality assumption is essential; indeed, the line arrangement $\mathcal{A}$ : $x^{2}+y^{2}=0$ is defined over $\mathbb{Q}(i)$, and the corresponding complement $M(\mathcal{A})$ satisfies $\left|M(\mathcal{A})\left(\mathbb{F}_{p}\right)\right|=p-1$ if $p \equiv 1 \bmod 4$ and $\left|M(\mathcal{A})\left(\mathbb{F}_{p}\right)\right|=p+1$ if $p \equiv 3$ $\bmod 4$. Note that in this case $Q(x)$ has integer coefficients and $\mathcal{A}$ is reducible, but the corresponding splitting $\mathcal{A}=\mathcal{A}_{1} \times \mathcal{A}_{2}$ is not defined over $\mathbb{Q}$.

A theorem of Katz in [11] says that if $Y$ has polynomial count with count polynomial $P_{Y}$, then $H D_{Y}(x, y)=P_{Y}(x y)$ (see [11, Theorem 2.1.8, the remark at the bottom of p. 563, and Example 2.1.10] explaining that there can be allowed finitely many "bad characteristics" $p$ ). In particular, such a variety is not too far from being cohomologically Tate; that is, the not-Tate part of the cohomology should cancel out in $H D_{Y}(x, y)$.

One may ask what happens to the Milnor fibers of hyperplane arrangements. Again, we need a rationality assumption: the Milnor fiber $F: x^{2}+$ $y^{2}=1$ satisfies $\left|F\left(\mathbb{F}_{p}\right)\right|=p-1$ if $p \equiv 1 \bmod 4$ and $\left|F\left(\mathbb{F}_{p}\right)\right|=p+1$ if $p \equiv 3$ $\bmod 4$. Note that in this example the monodromy operator $h^{*}$ is trivial and $F$ is cohomologically Tate.

When $\mathcal{A}$ is defined over $\mathbb{Q}$, we will always choose the defining equation $Q(x)=0$ with integer coefficients. It turns out that the arithmetic properties of the corresponding Milnor fiber $F: Q(x)-1=0$ do not depend on the choice of $Q$ (see, e.g., Corollary 1.7).

So a first naive idea when trying to construct cohomologically Tate Milnor fibers of rational arrangements which have no polynomial count is to look for a hyperplane arrangement $\mathcal{A}$ with a trivial monodromy $h^{*}$ and such that the corresponding Milnor fiber $F$ has no polynomial count. Such an attempt cannot succeed in view of the following result, implied by Theorem 1.2.

COROLlary 1.7. If the monodromy action $h^{*}$ is trivial on all the cohomology groups $H^{*}(F, \mathbb{C})$ of the Milnor fiber $F$ of an essential central arrangement $\mathcal{A}$ defined over $\mathbb{Q}$, then $F$ has a polynomial count, with the same count polynomial as the corresponding projective complement $M(\mathcal{A})$.

In particular, this property of the Milnor fiber having a polynomial count, and the corresponding count polynomial, does not depend on the choice of defining equation $Q$ in $\mathbb{Z}[x]$.

For $n=2$ we have the following.

COROllary 1.8. Consider the following conditions. 
(i) $Y$ has a polynomial count.

(ii) $Y$ is cohomologically Tate.

If $Y$ is a smooth affine surface, then (i) $\Longrightarrow$ (ii). If, in addition, $Y$ is the Milnor fiber of a central rational plane arrangement in $\mathbb{C}^{3}$, then one also has (ii) $\Longrightarrow$ (i).

The hyperplane arrangement $\mathcal{A}_{1,1}$ introduced in Example 4.3 is defined over $\mathbb{Q}$, and the corresponding Milnor fiber is cohomologically Tate but has no polynomial count (see Theorem 5.2).

\section{§2. Proof of Theorem 1.4 and of Corollary 1.7}

Proof. We can, up to a linear coordinate change, write $Q(x)=Q_{1}\left(y_{1}\right) \ldots$ $Q_{q}\left(y_{q}\right)$, where $x=\left(y_{1}, \ldots, y_{q}\right) \in \mathbb{C}^{n+1}, y_{j} \in \mathbb{C}^{n_{j}}$ such that $n_{1}+\cdots+n_{q}=$ $n+1, d_{j}=\operatorname{deg} Q_{j}>0$, and $\mathcal{A}_{j}: Q_{j}=0$ is an irreducible and essential arrangement in $\mathbb{C}^{n_{j}}$ for $j=1, \ldots, q$.

Note that the existence of such a decomposition is equivalent to the following property: there is a partition of the hyperplanes in $\mathcal{A}$ in $q$ subsets $A_{1}, \ldots, A_{q}$ such that if we define $V_{j}=\bigcap_{H \notin A_{j}} H$, then there is a direct sum decomposition $\mathbb{C}^{n+1}=V_{1}+V_{2}+\cdots+V_{q}$, and this partition is the finest with this property. More precisely, we have the following result.

Lemma 2.1. Let $\mathcal{A}=\left\{H_{i}\right\}_{i \in I}$ be a central, essential hyperplane arrangement in $\mathbb{C}^{n+1}=V$. Consider the set $P$ of partitions $I=I_{1} \cup \cdots \cup I_{m}$ of $I$ satisfying the following condition: $\sum_{j} \operatorname{codim}\left(\bigcap_{i \in I_{j}} H_{i}\right)=\operatorname{dim} V$. Then the following hold.

(i) $P$ is nonempty, since the trivial partition $I=I$ is in $P$.

(ii) If $I=I_{1} \cup \cdots \cup I_{m}$ and $I=I_{1}^{\prime} \cup \cdots \cup I_{m^{\prime}}^{\prime}$ are two partitions in $P$, then their intersection $I=\bigcup_{i, j} I_{i, j}$, where $I_{i, j}=I_{i} \cap I_{j}$ for $i=1, m, j=1, m^{\prime}$ (the empty intersections $I_{i, j}$ are discarded), is again a partition in $P$.

(iii) In the (unique) decomposition $\mathcal{A}=\mathcal{A}_{1} \times \cdots \times \mathcal{A}_{q}$ of $\mathcal{A}$ as a product of irreducible arrangements $\mathcal{A}_{j}$, the integers $q, d_{1}, \ldots, d_{q}$ are determined by the combinatorics, that is, by the intersection lattice $L(\mathcal{A})$.

Proof. Claim (i) is obvious.

Let $E$ be the dual of $V$, and, for a given partition $I=I_{1} \cup \cdots \cup I_{m}$ in $P$, let $E_{i}$ be the vector subspace in $E$ spanned by the equations of the hyperplanes in $I_{i}$. Since $\mathcal{A}$ is essential, it follows that $\sum_{j} E_{j}=E$. Since $\operatorname{dim} E_{j}=\operatorname{codim}\left(\bigcap_{i \in I_{j}} H_{i}\right)$, it follows that the above sum is in fact a direct sum. 
When we have two partitions as above, we define $E_{i, j}$ to be the vector subspace in $E$ spanned by the equations of the hyperplanes in $I_{i} \cap I_{j}^{\prime}$. Then it follows immediately that the sum $\sum_{j} E_{i, j}=E_{i}$ is again direct sum for all $i$. Hence, the sum $\sum_{i, j} E_{i, j}=V$ is a direct sum, which proves claim (ii).

Since the set of partitions $P$ is defined only in terms of the intersection lattice $L(\mathcal{A})$, it follows that the unique minimal element of $P$ (with respect to the partial order given by refinement) is combinatorially determined. This minimal element is denoted above by $I=A_{1} \cup \cdots \cup A_{q}$. The direct sum decomposition $\mathbb{C}^{n+1}=V_{1}+V_{2}+\cdots+V_{q}$ mentioned above is just the dual of the direct sum decomposition $\sum_{j} E_{j}=E$. Since $d_{j}=\left|A_{j}\right|$, they are also determined by the combinatorics.

Remark 2.2. When $\mathcal{A}$ is defined over $\mathbb{Q}$, it follows that all the vector subspaces $V_{j}$ are defined over $\mathbb{Q}$. Then the arrangement $\mathcal{A}_{j}$, which is essentially given by the traces of $H \in A_{j}$ on $V_{j}$, is clearly defined over $\mathbb{Q}$. Moreover, the coordinate change from the coordinates $x$ to the coordinates $y$ is defined over $\mathbb{Q}$. This shows that when doing computations for almost all primes $p$, we may replace the equation $Q(x)=0$ (resp., $Q(x)=1$ ) by the corresponding equations $Q_{1}\left(y_{1}\right) \cdots Q_{q}\left(y_{q}\right)=0$ (resp., $Q_{1}\left(y_{1}\right) \cdots Q_{q}\left(y_{q}\right)=1$ ).

Proof of Theorem 1.4. Let $F_{j}: Q_{j}=1$ and $h_{j}: F_{j} \rightarrow F_{j}$ be the corresponding Milnor fibers and monodromy homeomorphisms. Let us consider the corresponding least common multiple $m=\operatorname{LCM}\left(d_{1}, \ldots, d_{q}\right)$ and set $w_{j}=$ $m / d_{j}$ for $j=1, \ldots, q$.

Our first aim is to obtain a description of the (total) Milnor fiber $F$ in terms of the collection of Milnor fibers $F_{1}, \ldots, F_{q}$. For this we consider the affine torus

$$
\mathbb{T}=\left\{t=\left(t_{1}, \ldots, t_{q}\right) \in\left(\mathbb{C}^{*}\right)^{q} \mid t_{1} t_{2} \cdots t_{q}=1\right\} .
$$

Consider the mapping

$$
f: \mathbb{T} \times F_{1} \times \cdots \times F_{q} \rightarrow F
$$

given by

$$
\left(t, y_{1}, \ldots, y_{q}\right) \mapsto\left(t_{1}^{w_{1}} y_{1}, \ldots, t_{q}^{w_{q}} y_{q}\right)
$$

It is easy to check that this mapping $f$ is surjective and that one has

$$
f\left(t, y_{1}, \ldots, y_{q}\right)=f\left(t^{\prime}, y_{1}^{\prime}, \ldots, y_{q}^{\prime}\right)
$$


if and only if the points $\left(t, y_{1}, \ldots, y_{q}\right)$ and $\left(t^{\prime}, y_{1}^{\prime}, \ldots, y_{q}^{\prime}\right)$ are in the same $G$-orbit, where the group

$$
G=\left\{g=\left(g_{1}, \ldots, g_{q}\right) \in \mu_{m}^{q} \mid g_{1} g_{2} \cdots g_{q}=1\right\}
$$

acts on $X=\mathbb{T} \times F_{1} \times \cdots \times F_{q}$ via

$$
g \cdot\left(\left(t_{1}, \ldots, t_{q}\right), y_{1}, \ldots, y_{q}\right)=\left(\left(g_{1}^{-1} t_{1}, \ldots, g_{q}^{-1} t_{q}\right), g_{1}^{w_{1}} y_{1}, \ldots, g_{q}^{w_{q}} y_{q}\right) .
$$

It follows that $F=X / G$ and, in particular, that $H^{*}(F, \mathbb{Q})=H^{*}(X, \mathbb{Q})^{G}$, the $G$-fixed part of the cohomology of $X$ under the induced $G$-action. This is an isomorphism of MHS, since the $G$-action is algebraic. Note that

$$
H^{*}(X, \mathbb{C})=H^{*}(\mathbb{T}, \mathbb{C}) \otimes H^{*}\left(F_{1}, \mathbb{C}\right) \otimes \cdots \otimes H^{*}\left(F_{q}, \mathbb{C}\right) .
$$

Moreover, the group $G$ acts trivially on the factor $H^{*}(\mathbb{T}, \mathbb{C})$, since $\mathbb{T}$ is a connected algebraic group and $G \subset \mathbb{T}$. If we set

$$
H^{*}=H^{*}\left(F_{1}, \mathbb{R}\right) \otimes \cdots \otimes H^{*}\left(F_{q}, \mathbb{R}\right),
$$

then it follows that

$$
H^{*}(F, \mathbb{R})=H^{*}(\mathbb{T}, \mathbb{R}) \otimes\left(H^{*}\right)^{G} .
$$

The $G$-action on $H_{\mathbb{C}}^{*}$, the complexification of $H^{*}$, is given by the following: if $\eta=\eta_{1} \otimes \cdots \otimes \eta_{q}$, then

$$
g \eta=\left(h_{1}\right)^{k_{1}}\left(\eta_{1}\right) \otimes \cdots \otimes\left(h_{q}\right)^{k_{q}}\left(\eta_{q}\right) .
$$

Here $g=\left(\lambda^{k_{1}}, \ldots, \lambda^{k_{q}}\right)$ with $\lambda=\exp (2 \pi i / m)$ and $k_{1}+\cdots+k_{q}$ is divisible by $m$, the $k_{j}$ being otherwise arbitrary integers.

Let $\eta_{j} \in H^{*}\left(F_{j}, \mathbb{C}\right)$ be now chosen such that for any $j=1, \ldots, q$ there is a $\beta_{j} \in \mu_{d_{j}} \subset \mu_{m}$ with $h_{j}^{*} \eta_{j}=\beta_{j} \eta_{j}$, and look at $\eta=\eta_{1} \otimes \cdots \otimes \eta_{q}$. Such elements form a $\mathbb{C}$-basis of $H_{\mathbb{C}}^{*}$, and hence to determine $\left(H_{\mathbb{C}}^{*}\right)^{G}$ is the same as finding all $\eta$ of this form which are fixed under the $G$-action. By choosing $k_{q}=m-k_{1}-\cdots-k_{q-1}$, we get from (2.7) the following:

$$
g \eta=\left(\frac{\beta_{1}}{\beta_{q}}\right)^{k_{1}} \cdots\left(\frac{\beta_{q-1}}{\beta_{q}}\right)^{k_{q-1}} \eta,
$$

where now there is no condition on the integers $k_{1}, \ldots, k_{q-1}$. By taking one of them equal to 1 and the rest zero, we see that $\eta \in\left(H_{\mathbb{C}}^{*}\right)^{G}$ implies that $\beta_{1}=$ $\cdots=\beta_{q}$. Call this common value $\lambda_{0}$, and note that $\lambda_{0} \in \mu_{d_{0}}=\bigcap_{j=1, q} \mu_{d_{j}}$. 
Conversely, for any $\lambda_{0} \in \mu_{d_{0}}$ and any $\eta \in H^{*}\left(F_{1}, \mathbb{C}\right)_{\lambda_{0}} \otimes \cdots \otimes H^{*}\left(F_{q}, \mathbb{C}\right)_{\lambda_{0}}$, we see by using $(2.7)$ that $\eta \in\left(H_{\mathbb{C}}^{*}\right)^{G}$.

Moreover, set $E_{\lambda_{0}}=H^{*}\left(F_{1}, \mathbb{C}\right)_{\lambda_{0}} \otimes \cdots \otimes H^{*}\left(F_{q}, \mathbb{C}\right)_{\lambda_{0}}$, and note that $\bar{E}_{\lambda_{0}}=$ $E_{\bar{\lambda}_{0}}$.

It follows that if we set $M_{\lambda_{0}}=E_{\lambda_{0}}$ when $\lambda_{0} \in \mathbb{R}$ and $M_{\lambda_{0}}=E_{\lambda_{0}}+E_{\bar{\lambda}_{0}}$ when $\lambda_{0} \notin \mathbb{R}$, then $M_{\lambda_{0}}$ is endowed with a natural $\mathbb{R}$-MHS (see also (4.1)).

To prove the second part of Theorem 1.4(i), we construct a nice $G$ equivariant lifting $\tilde{h}: X \rightarrow X$ of the monodromy morphism $h: F \rightarrow F$. We set

$$
\tilde{h}\left(t_{1}, \ldots, t_{q}, y_{1}, \ldots, y_{q}\right)=\left(\gamma_{1} t_{1}, \ldots, \gamma_{q} t_{q}, \beta_{1} y_{1}, y_{2}, \ldots, y_{q}\right),
$$

where $\beta_{1}=\exp \left(2 \pi i / d_{1}\right)$ and $\gamma_{j}=\exp \left(2 \pi i a_{j}\right)$ with $w_{1} a_{1}=1 / d-1 / d_{1}$ and $w_{j} a j=1 / d$ for $j>1$. Then $\sum_{j} a_{j}=0$; that is, $\left(\gamma_{1} t_{1}, \ldots, \gamma_{q} t_{q}\right) \in \mathbb{T}$, and $f \circ \tilde{h}=h \circ f$. Then $\tilde{h}^{*}$ acts as identity on all the factors in the tensor product (2.6), except on $H^{*}\left(F_{1}, \mathbb{C}\right)$, where it acts via $h_{1}^{*}$. We get the second part of claim (i) by using the description of the cohomology $H^{*}(F, \mathbb{C})$ given in the first part of claim (i).

To prove claim (ii), note that the affine torus $\mathbb{T}$ acts on the Milnor fiber $F$ by

$$
t\left(y_{1}, \ldots, y_{q}\right)=\left(t_{1}^{w_{1}} y_{1}, \ldots, t_{q}^{w_{q}} y_{q}\right) .
$$

Hence, to show that $\left(h^{*}\right)^{d_{0}}$ is trivial, it is enough to show the existence of an element $t=\left(t_{1}, \ldots, t_{q}\right) \in \mathbb{T}$ such that $t_{j}^{w_{j}}=\exp \left(2 \pi i d_{0} / d\right)$ for $j=1, \ldots, q$. Since $\operatorname{GCD}\left(d_{1}, \ldots, d_{q}\right)=d_{0}$, there are integers $k_{j}$ such that

$$
k_{1} d_{1}+\cdots+k_{q} d_{q}=(m-1) d_{0} .
$$

For $j=1, \ldots, q$, we set

$$
t_{j}=\exp \left[2 \pi i\left(\frac{d_{0}}{d w_{j}}+\frac{k_{j}}{w_{j}}\right)\right] .
$$

The relations $t_{j}^{w_{j}}=\exp \left(2 \pi i d_{0} / d\right)$ are clearly satisfied. Moreover,

$t_{1} t_{2} \cdots t_{q}=\exp \left[2 \pi i\left(\sum_{j}\left(\frac{d_{0} d_{j}}{d m}+\frac{k_{j} d_{j}}{m}\right)\right)\right]=\exp \left[2 \pi i d_{0}\left(\frac{1}{m}+\frac{m-1}{m}\right)\right]=1$.

Hence, $t \in \mathbb{T}$, so it follows that $\left(h^{*}\right)^{d_{0}}$ is trivial. We conclude using the following fact: since each $\mathcal{A}_{j}$ is irreducible, it follows from (1.2) that each $H^{*}\left(F_{j}\right)_{\beta}$ which occurs in Theorem 1.4 is nonzero. Hence, each $H^{*}(F)_{\beta}$ is nonzero; that is, the order of $h^{*}$ is indeed $d_{0}$. 
REMARK 2.3. Consider the central essential hyperplane arrangement $\mathcal{A}$ in $\mathbb{C}^{n+1}$ and its decomposition $\mathcal{A}=\mathcal{A}_{1} \times \cdots \times \mathcal{A}_{q}$ as a product of irreducible arrangements $\mathcal{A}_{j}$ for $j=1, \ldots, q$. Then it is easy to show that

$$
M(\mathcal{A})=\mathbb{T} \times M\left(\mathcal{A}_{1}\right) \times \cdots \times M\left(\mathcal{A}_{q}\right) .
$$

This implies the following for the cohomology of the corresponding projective complements:

$$
H^{*}(M(\mathcal{A}))=H^{*}\left(\mathbb{C}^{*}\right)^{\otimes(q-1)} \otimes H^{*}\left(M\left(\mathcal{A}_{1}\right)\right) \otimes \cdots \otimes H^{*}\left(M\left(\mathcal{A}_{q}\right)\right)
$$

that is, the case $\beta=1$ in Theorem 1.4(iii) (see also [29]).

Now we pass to the proof of Corollary 1.7. Since the monodromy $h^{*}$ is trivial, we know by Theorem 1.2 that $\operatorname{GCD}\left(d_{1}, \ldots, d_{q}\right)=1$. Hence, there exist integers $m_{j}$ such that $m_{1} d_{1}+\cdots+m_{q} d_{q}=1$.

Let $\mathbb{F}$ be a finite field, and consider the mapping $Q: \mathbb{F}^{n+1} \rightarrow \mathbb{F}$ induced by the polynomial $Q$.

For $a \in \mathbb{F}$, denote by $F(a)$ the fiber $Q^{-1}(a)$. Denote also $M(\mathcal{A}, \mathbb{F})$ the corresponding (projective) hyperplane arrangement complement over $\mathbb{F}$. It is clear that

$$
\left|\mathbb{F}^{n+1} \backslash F(0)\right|=(|\mathbb{F}|-1) \cdot|M(\mathcal{A}, \mathbb{F})|
$$

Consider the following $\mathbb{F}^{*}$-action on $\mathbb{F}^{n+1}$ :

$$
t \cdot x=t \cdot\left(y_{1}, y_{2}, \ldots, y_{q}\right)=\left(t^{m_{1}} y_{1}, t^{m_{2}} y_{2}, \ldots, t^{m_{q}} y_{q}\right)
$$

The relation $Q(t \cdot x)=t Q(x)$ shows that all the fibers $F(a)$ for $a \in \mathbb{F}^{*}$ have the same cardinal. Since their disjoint union is exactly $\mathbb{F}^{n+1} \backslash F(0),(2.12)$ yields

$$
|F(1)|=|M(\mathcal{A}, \mathbb{F})|
$$

This equality completes the proof of Corollary 1.7.

\section{§3. Proof of Theorem 1.1 and of Corollary 1.8}

Proof. It follows from the discussion just after Theorem 1.1 that, assuming Theorem 1.1(ii), the Milnor fiber $F$ is isomorphic to the complement of the central line arrangement given by $Q_{1}=0$ in $\mathbb{C}^{2}$. (Indeed, the only 
partitions of 3 are $1+2=2+1=1+1+1=3$.) Hence, the implication (ii) $\Rightarrow$ (i) is obviously true.

Note that for $n=2$ and $\alpha \in(0,1)$, the corresponding spectral number is by definition

$$
m_{\alpha}=h^{2,0}\left(H^{2}(F, \mathbb{C})_{\beta}\right)+h^{2,1}\left(H^{2}(F, \mathbb{C})_{\beta}\right)
$$

with $\beta=\exp (-2 \pi i \alpha)$. Indeed, $h^{2,2}\left(H^{2}(F, \mathbb{C})_{\beta}\right)=0$, as follows from [8, Theorem 1.3].

Since $H^{2}(F, \mathbb{C})_{1}$ is known to be of type $(2,2)$, the equivalence of Theorem 1.1(i), (iv) follows. Moreover, the equivalence of claims (ii) and (iii) follows from Theorem 1.2, using again the partitions of 3 .

So from now on we assume that Theorem 1.1(iv) holds, and we prove (ii). The fact that the arrangement is essential implies that $d \geq 3$ and that the lines in $\mathcal{A}^{\prime}$ do not pass all through the same point; that is, there is no point $s$ of multiplicity $m_{s}=d$. To prove (ii) we have to show the existence of a point of multiplicity $d-1$.

For $d=3$ there are only two types of arrangements, described better in terms of their associated projective line arrangements $\mathcal{A}^{\prime}$ :

(a) three lines forming a triangle, in which case one may take $Q=x y z$ and $F=\mathbb{C}^{*} \times \mathbb{C}^{*}$, and

(b) three lines meeting at one point.

The claim (i) $\Rightarrow$ (ii) is clear by the previous remark.

We assume from now on that $d \geq 4$.

Next we recall the following key formula from [1, Theorem 3] (rewritten slightly for our needs). If $0<\alpha=j / d<1$, then

$$
m_{\alpha}=\left(\begin{array}{c}
j-1 \\
2
\end{array}\right)-\sum_{s ; m_{s} \geq 3}\left(\begin{array}{c}
\left\lceil j m_{s} / d\right\rceil-1 \\
2
\end{array}\right),
$$

where the sum is over all multiple points $s$ in $\mathcal{A}^{\prime}$ with multiplicity $m_{s} \geq 3$. By convention, $\left(\begin{array}{l}a \\ b\end{array}\right)=0$ if $a<b$.

If we use the above formula for $j=3$, we get that the corresponding vanishing $m_{\alpha}=0$ is equivalent to the existence of a unique point $s$ of multiplicity $m_{s}>2 d / 3$ in $\mathcal{A}^{\prime}$. For $d=4$ (resp., $d=5$ ), this means a point of multiplicity $m_{s} \geq 3$ (resp., $m_{s} \geq 4$ ). As above (case $d=3$, (b)), the case $m_{s}=4$ (resp., $m_{s}=5$ ) is discarded since $\mathcal{A}^{\prime}$ is essential. Hence, $m_{s}=3$ (resp., $m_{s}=4$ ), which gives exactly an arrangement $\mathcal{A}^{\prime}$ as claimed in (ii). 
From now on we assume that $d>5$. We apply (3.2) for $j=d-1$. Since one clearly has

$$
m-1<\frac{(d-1) m}{d}<m,
$$

it follows that $\lceil(d-1) m / d\rceil=m$, and hence the vanishing $m_{\alpha}=0$ in this case is equivalent to the equality

$$
\left(\begin{array}{c}
d-2 \\
2
\end{array}\right)=\sum_{s ; m_{s} \geq 3}\left(\begin{array}{c}
m_{s}-1 \\
2
\end{array}\right)
$$

Similarly, for $j=d-2$ we get from $m_{\alpha}=0$ the equality

$$
\left(\begin{array}{c}
d-3 \\
2
\end{array}\right)=\sum_{s ; 3 \leq m_{s}<d / 2}\left(\begin{array}{c}
m_{s}-1 \\
2
\end{array}\right)+\sum_{s ; m_{s} \geq d / 2}\left(\begin{array}{c}
m_{s}-2 \\
2
\end{array}\right) .
$$

By taking the difference of (3.3) and (3.4), we get the equality

$$
d-3=\sum_{s ; m_{s} \geq d / 2}\left(m_{s}-2\right)
$$

It follows that, if the set $S=\left\{s ; m_{s} \geq d / 2\right\}$ contains exactly one element, then the corresponding multiple point $s$ satisfies $m_{s}=d-1$, and we are done.

Suppose now that the set $S$ contains at least two elements. Since one of them has to be the point $s$ with multiplicity $m_{s}>2 d / 3$ obtained above for $j=3$, we get in this case

$$
d-3>(2 d / 3-2)+(d / 2-2) \text {. }
$$

This is equivalent to $d<6$, a contradiction with our hypothesis $d>5$.

The proof of Corollary 1.8 is very easy now. If Corollary 1.8(i) holds, then it follows from Katz's theorem that the Hodge-Deligne polynomial of $Y$ contains only the monomials $1, x y,(x y)^{2}$. The possible nonzero mixed Hodge numbers in this situation are

$$
\begin{aligned}
& h^{2,2}\left(H_{c}^{4}(Y, \mathbb{C})\right), h^{1,2}\left(H_{c}^{3}(Y, \mathbb{C})\right), h^{2,1}\left(H_{c}^{3}(Y, \mathbb{C})\right), \\
& h^{1,1}\left(H_{c}^{3}(Y, \mathbb{C})\right), h^{2,0}\left(H_{c}^{2}(Y, \mathbb{C})\right), \\
& h^{1,1}\left(H_{c}^{2}(Y, \mathbb{C})\right), h^{0,2}\left(H_{c}^{2}(Y, \mathbb{C})\right), h^{0,1}\left(H_{c}^{2}(Y, \mathbb{C})\right),
\end{aligned}
$$




$$
h^{1,0}\left(H_{c}^{2}(Y, \mathbb{C})\right), h^{0,0}\left(H_{c}^{2}(Y, \mathbb{C})\right)
$$

It follows that the only cancellations in the Hodge polynomial can occur in the coefficient of $x y$. Hence, all the mixed Hodge numbers $h^{u, v}\left(H_{c}^{m}(Y, \mathbb{C})\right)$ vanish for $u \neq v$; that is, $Y$ is cohomologically Tate.

Now for Milnor fibers of a central plane arrangement, we have seen that Theorem 1.1(ii) implies that $Y$ is isomorphic to a line arrangement complement in $\mathbb{C}^{2}$, and hence it has polynomial count.

\section{$\S 4$. A purity result and the key example}

Let $\mathcal{A}$ be a central arrangement of $d$ hyperplanes in $\mathbb{C}^{n+1}$, with $n \geq$ 1 , given by a reduced equation $Q(x)=0$. Then clearly $H^{n}(F, \mathbb{Q})_{1}$ and $H^{n}(F, \mathbb{C})_{-1}$ are mixed Hodge substructures in $H^{n}(F, \mathbb{Q})$. Moreover, for $\beta \in \mu_{d}, \beta \neq \pm 1$, the same is true for the subspace

$$
H^{n}(F, \mathbb{C})_{\beta, \bar{\beta}}=H^{n}(F, \mathbb{C})_{\beta} \oplus H^{n}(F, \mathbb{C})_{\bar{\beta}}=\operatorname{ker}\left[\left(h^{n}\right)^{2}-2 \operatorname{Re}(\beta) h^{n}+I d\right],
$$

which is in fact defined over $\mathbb{R}$ (as the last equality shows). For $\beta=-1$, we set $H^{n}(F, \mathbb{C})_{\beta, \bar{\beta}}=H^{n}(F, \mathbb{C})_{-1}$ for uniformity of notation.

Let

$$
D=Q^{-1}(0)=\bigcup_{H \in \mathcal{A}} H .
$$

For a point $x \in D, x \neq 0$, let $L_{x}=\bigcap_{H \in \mathcal{A}, x \in H} H$, and denote by $\mathcal{A}_{x}$ the central hyperplane arrangement induced by $\mathcal{A}$ on a linear subspace $T_{x}$, passing through $x$ and transversal to $L_{x}$. We may choose $\operatorname{dim} T_{x}=\operatorname{codim} L_{x}$ and identify $x$ with the origin in the linear space $T_{x}$. Let $h_{x}^{*}: H^{*}\left(F_{x}, \mathbb{C}\right) \rightarrow$ $H^{*}\left(F_{x}, \mathbb{C}\right)$ be the corresponding monodromy operator at $x$.

With this notation, we have the following result.

Proposition 4.1. Let $\beta \in \mu_{d}, \beta \neq 1$ be a root of unity which is not an eigenvalue for any monodromy operator $h_{x}^{*}$ for $x \in D, x \neq 0$. Then the corresponding eigenspace $H^{n}(F, \mathbb{C})_{\beta, \bar{\beta}}$ is a pure Hodge structure of weight $n$.

In particular, if $\beta=\exp (-2 \pi i \alpha)$ for some $\alpha \in \mathbb{Q}$, then the coefficients in the corresponding spectrum $\operatorname{Sp}(\mathcal{A})$ have the following symmetry property:

$$
m_{\alpha}=m_{n+1-\alpha} .
$$

Proof. This result is a direct consequence of [22, Lemma 3.6]. Indeed, our hypothesis on $\beta$ implies that the nearby cycle sheaf $\psi_{Q, \beta} \mathbb{C}$ is supported at 
the origin, and hence it is identified to $H^{n}(F, \mathbb{C})_{\beta}$. This identification in turn implies that the logarithm of the unipotent part of the monodromy $N$ is trivial on $\psi_{Q, \beta} \mathbb{C}$ (as this holds for $H^{n}(F, \mathbb{C})_{\beta}$, the monodromy $h^{*}$ being semisimple).

The last claim about the symmetry property in (4.2) is proved in the usual way. In view of the purity result, we have $h^{p, q}\left(H^{n}(F, \mathbb{C})_{\beta}\right)=0$ for $p+q \neq n$.

For example, assume that $\alpha=j / d$ with $0<j<d$. It follows from the definition of the spectrum $(1.1)$ that $m_{\alpha}=\operatorname{dim} h^{n, 0}\left(H^{n}(F, \mathbb{C})_{\beta}\right), m_{n+1-\alpha}=$ $\operatorname{dim} h^{0, n}\left(H^{n}(F, \mathbb{C})_{\bar{\beta}}\right)$. The claimed equality follows by considering the action of the complex conjugation on the pure Hodge structure $H^{n}(F, \mathbb{C})_{\beta, \bar{\beta}}$.

Corollary 4.2. Assume that $\mathcal{A}$ is a generic central hyperplane arrangement; that is, the associated projective divisor in $\mathbb{P}^{n}$ is a divisor with normal crossing. Then

$$
H^{n}(F, \mathbb{C})_{\neq 1}=\bigoplus_{\beta \neq 1} H^{n}(F, \mathbb{C})_{\beta}
$$

is a pure Hodge structure of weight $n$, and for any rational number $\alpha \in \mathbb{Q} \backslash \mathbb{Z}$ one has $m_{\alpha}=m_{n+1-\alpha}$.

This symmetry of the coefficients of the $\operatorname{spectrum} \operatorname{Sp}(\mathcal{A})$ of a generic central arrangement is alluded to in [22] (see the remarks just after Corollary 1 in the introduction). The result above follows from Proposition $4.1 \mathrm{using}$ the simple fact that in this case all the monodromy operators $h_{x}^{*}$ are the identity. For more on the monodromy of generic arrangements, see [21, pp. 209-210], [1, (3.2)], and [3, Section 3].

Now we pass to our example.

EXAMPLE 4.3 . For $n \geq 2$, let $\mathcal{G}_{n}$ be the central arrangement in $\mathbb{C}^{n+1}$ given by the equation

$$
Q_{n}(x)=Q_{n}\left(x_{0}, \ldots, x_{n}\right)=x_{0} \cdot x_{1} \cdots x_{n} \cdot\left(x_{0}+x_{1}+\cdots+x_{n}\right) .
$$

Hence, the degree $d_{n}$ of $Q_{n}$ is $n+2$, and clearly $\mathcal{G}_{n}$ is a generic, irreducible arrangement. Assume that $n=2 k$ is even, and use [3, Theorem 3.2] (or refer to the original paper [19]) to get that

$$
H^{m}(F, \mathbb{C})=H^{m}(F, \mathbb{C})_{1}
$$

for $0 \leq m<n$ and $\operatorname{dim} H^{n}(F, \mathbb{C})_{-1}=1$ 
It follows that all the spectral coefficients in $\operatorname{Sp}\left(\mathcal{G}_{n}\right)$ corresponding to the monodromy eigenvalue -1 vanish except for $m_{k+(1 / 2)}=1$ (which is the only autodual element in the sum with respect to the symmetry given by Corollary 4.2). It follows that the eigenspace $H^{n}(F, \mathbb{C})_{-1}$ is spanned by a cohomology class $\omega_{n}$ of Hodge type $(k, k)$.

Let us return now to the setting of the proof of Theorem 1.2 in Section 2. Let $\mathcal{A}_{u, v}$ be the central hyperplane arrangement obtained by taking the product of $u>0$ copies of the arrangement $\mathcal{G}_{2}$ and $v>0$ copies of $\mathcal{G}_{4}$. It follows that $n=3 u+5 v-1, d=4 u+6 v, q=u+v$, and $d_{0}=\operatorname{GCD}\left(d_{1}, \ldots\right.$, $\left.d_{q}\right)=2$.

In this case, the cohomology of the corresponding total Milnor fiber $F_{u, v}$ can be described via Theorem 1.4 as the direct sum $H^{*}\left(F_{u, v}, \mathbb{C}\right)=$ $H^{*}\left(F_{u, v}, \mathbb{C}\right)_{1} \oplus H^{*}\left(F_{u, v}, \mathbb{C}\right)_{-1}$, where

$$
H^{*}\left(F_{u, v}, \mathbb{C}\right)_{1}=H^{*}(\mathbb{T}, \mathbb{C}) \otimes H^{*}\left(M\left(\mathcal{G}_{2}\right), \mathbb{C}\right)^{\otimes u} \otimes H^{*}\left(M\left(\mathcal{G}_{4}\right), \mathbb{C}\right)^{\otimes v}
$$

and

$$
H^{*}\left(F_{u, v}, \mathbb{C}\right)_{-1}=H^{*}(\mathbb{T}, \mathbb{C}) \otimes\left(\mathbb{C} \omega_{2}\right)^{\otimes u} \otimes\left(\mathbb{C} \omega_{4}\right)^{\otimes v}
$$

It follows that $F_{u, v}$ is a cohomologically Tate variety of dimension $n=$ $3 u+5 v-1$, but the corresponding monodromy action $h^{*}$ is not trivial. By choosing various values for $u, v$, we can get $n=7$ as a minimal value as well as any integer $n \geq 15$.

REMARK 4.4. If one is interested only in irreducible arrangements, then such examples with large cohomology $H^{<\operatorname{top}}(F, \mathbb{C})$ can be obtained by taking a generic hyperplane section of the arrangement $\mathcal{A}_{u, v}$.

\section{$\S 5 . \quad$ Finite field computations}

In this section we use the following notation. Let $F=F_{1,1}$ be the variety defined over $\mathbb{Z}$ by $Q(x)=1$, where

$Q(x)=x_{1} \cdot x_{2} \cdot x_{3} \cdot\left(x_{1}+x_{2}+x_{3}\right) \cdot x_{4} \cdot x_{5} \cdot x_{6} \cdot x_{7} \cdot x_{8} \cdot\left(x_{4}+x_{5}+x_{6}+x_{7}+x_{8}\right)$.

For any prime $p$, we denote by $A(p)$ the number of points of $F$ over $\mathbb{F}_{p}=$ $\mathbb{Z} / p \mathbb{Z}$; that is, the number of solutions of the equation $Q(x)=1$ in $\mathbb{F}_{p}^{8}$. For $a \in \mathbb{F}_{p}^{*}$, consider the varieties

$$
F_{1}(a)=\left\{\left(x_{1}, x_{2}, x_{3}\right) \in \mathbb{F}_{p}^{3} \mid x_{1} \cdot x_{2} \cdot x_{3} \cdot\left(x_{1}+x_{2}+x_{3}\right)=a\right\}
$$


and

$$
\begin{aligned}
F_{2}(a)=\{ & \left(x_{4}, x_{5}, x_{6}, x_{7}, x_{8}\right) \in \mathbb{F}_{p}^{5} \\
& \left.\mid a \cdot x_{4} \cdot x_{5} \cdot x_{6} \cdot x_{7} \cdot x_{8} \cdot\left(x_{4}+x_{5}+x_{6}+x_{7}+x_{8}\right)=1\right\} .
\end{aligned}
$$

Let $n_{1}(a)=\left|F_{1}(a)\right|$, let $n_{2}(a)=\left|F_{2}(a)\right|$, and note that obviously one has

$$
A(p)=\sum_{a \in \mathbb{F}_{p}^{*}} n_{1}(a) n_{2}(a) .
$$

From now on we assume that $p$ is a prime number in the arithmetic progression $12 b+11$, where $b \in \mathbb{N}$.

Lemma 5.1. Consider the group morphism $p(k): \mathbb{F}_{p}^{*} \rightarrow \mathbb{F}_{p}^{*}$ given by $t \mapsto t^{k}$. Then the following hold. $H$.

(i) There is an index 2 subgroup $H \subset \mathbb{F}_{p}^{*}$ such that $p(4)\left(\mathbb{F}_{p}^{*}\right)=p(6)\left(\mathbb{F}_{p}^{*}\right)=$

(ii) If $a$ and $a^{\prime}$ have the same class in $\mathbb{F}_{p}^{*} / H$, then $n_{1}(a)=n_{1}\left(a^{\prime}\right)$ and $n_{2}(a)=n_{2}\left(a^{\prime}\right)$.

Proof. Since the multiplicative group $\mathbb{F}_{p}^{*}$ is cyclic, of order $p-1=12 b+10$, it follows that to prove (i) it is enough to show that $p(4)\left(\mathbb{F}_{p}^{*}\right)$ and $p(6)\left(\mathbb{F}_{p}^{*}\right)$ have both cardinal $(p-1) / 2$. This is the same as proving that the corresponding kernels have order 2 . Note that the equation $t^{4}=1$ (resp., $t^{6}=1$ ) is equivalent to $t^{2}=1$ (look at the order of $t$, which must be a divisor of $12 b+10)$. Hence, $\operatorname{ker} p(4)=\operatorname{ker} p(6)=\{ \pm 1\}$ and hence have order 2 .

To prove (ii), consider the action of $\mathbb{F}_{p}^{*}$ on $\mathbb{F}_{p}^{3}$ (resp., $\mathbb{F}_{p}^{5}$ ) given by the usual multiplication of a vector by a scalar. This multiplication by $t \in \mathbb{F}_{p}^{*}$ induces a bijection $F_{1}(a)=F_{1}\left(t^{4} a\right)$ (resp., $F_{2}(a)=F_{2}\left(t^{6} a\right)$ ). This completes the proof.

We denote $n_{1}^{\prime}=n_{1}(1), n_{2}^{\prime}=n_{2}(1), n_{1}^{\prime \prime}=n_{1}(a)$, and $n_{2}^{\prime \prime}=n_{2}(a)$, for $a \in$ $\left(\mathbb{F}_{p}^{*} \backslash H\right)$. We also write $p=12 b+11=4 k+3$; that is, we set $k=3 b+2$. With this notation, (5.1) may be rewritten as

$$
A(p)=|H|\left(n_{1}^{\prime} n_{2}^{\prime}+n_{1}^{\prime \prime} n_{2}^{\prime \prime}\right)=(2 k+1)\left(n_{1}^{\prime} n_{2}^{\prime}+n_{1}^{\prime \prime} n_{2}^{\prime \prime}\right) .
$$

The (affine or projective) hyperplane arrangement complements have polynomial count (see, e.g., [7, Section (5.3)], [26, Theorem 5.15]). So we apply this fact to the projective hyperplane arrangement complements $\mathcal{A}_{1}^{\prime}$ 
in $\mathbb{P}^{2}$ (resp., $\mathcal{A}_{2}^{\prime}$ in $\mathbb{P}^{4}$ ) corresponding to $x_{1} x_{2} x_{3}\left(x_{1}+x_{2}+x_{3}\right)=0$ (resp., $\left.x_{4} x_{5} x_{6} x_{7} x_{8}\left(x_{4}+x_{5}+x_{6}+x_{7}+x_{8}\right)=0\right)$. Using the formulas for the Betti numbers in [3], the fact that each cohomology group $H^{m}$ is pure of type $(m, m)$, and the duality between $H^{m}$ and $H_{c}^{2 d-m}$ (where $d$ is the corresponding (complex) dimension), it follows that

$$
H D_{M\left(\mathcal{A}_{1}^{\prime}\right)}(x, y)=(x y)^{2}-3(x y)+3
$$

and

$$
H D_{M\left(\mathcal{A}_{2}^{\prime}\right)}(x, y)=(x y)^{4}-5(x y)^{3}+10(x y)^{2}-10(x y)+5 .
$$

It follows that the corresponding counting polynomials are

$$
P_{M\left(\mathcal{A}_{1}^{\prime}\right)}(t)=t^{2}-3 t+3
$$

and

$$
P_{M\left(\mathcal{A}_{2}^{\prime}\right)}(t)=t^{4}-5 t^{3}+10 t^{2}-10 t+5 \text {. }
$$

Using these two polynomials, it follows that, for almost all $p=4 k+3$, one has

$$
N_{1}^{\prime}(p)=\left|M\left(\mathcal{A}_{1}^{\prime}\right)\left(\mathbb{F}_{p}\right)\right|=p^{2}-3 p+3 \equiv 4 k+3 \bmod 8
$$

and

$$
N_{2}^{\prime}(p)=\left|M\left(\mathcal{A}_{2}^{\prime}\right)\left(\mathbb{F}_{p}\right)\right|=p^{4}-5 p^{3}+10 p^{2}-10 p+5 \equiv 4 k+3 \bmod 8 .
$$

Let $\mathcal{A}_{1}$ (resp., $\mathcal{A}_{2}$ ) denote the corresponding central hyperplane arrangements in $\mathbb{C}^{3}$ (resp., $\mathbb{C}^{5}$ ) and $M\left(\mathcal{A}_{1}\right)$ (resp., $M\left(\mathcal{A}_{2}\right)$ ) the associated affine complements. Then, using (5.7) and (5.8), we get

$$
N_{1}(p)=\left|M\left(\mathcal{A}_{1}\right)\left(\mathbb{F}_{p}\right)\right|=(p-1)\left(p^{2}-3 p+3\right) \equiv 4 k+6 \bmod 8
$$

and

$N_{2}(p)=\left|M\left(\mathcal{A}_{2}\right)\left(\mathbb{F}_{p}\right)\right|=(p-1)\left(p^{4}-5 p^{3}+10 p^{2}-10 p+5\right) \equiv 4 k+6 \bmod 8$.

Now, note that $M\left(\mathcal{A}_{1}\right)$ (resp., $M\left(\mathcal{A}_{2}\right)$ ) is the disjoint union of all the hypersurfaces $F_{1}(a)$ (resp., $\left.F_{2}(a)\right)$ for $a \in \mathbb{F}_{p}^{*}$. Lemma 5.1, (5.9), and (5.10) yield

$$
n_{1}^{\prime}+n_{1}^{\prime \prime}=2\left(p^{2}-3 p+3\right) \equiv 6 \bmod 8
$$


and

$$
n_{2}^{\prime}+n_{2}^{\prime \prime}=2\left(p^{4}-5 p^{3}+10 p^{2}-10 p+5\right) \equiv 6 \bmod 8 .
$$

It follows that $n_{1}^{\prime \prime} \equiv 6-n_{1}^{\prime} \bmod 8$ and $n_{2}^{\prime \prime} \equiv 6-n_{2}^{\prime} \bmod 8$. Using (5.2), we get

$$
A(p)=(2 k+1)\left(n_{1}^{\prime} n_{2}^{\prime}+n_{1}^{\prime \prime} n_{2}^{\prime \prime}\right) \equiv 2(2 k+1)\left(2+n_{1}^{\prime} n_{2}^{\prime}-3 n_{1}^{\prime}-3 n_{2}^{\prime}\right) \bmod 8 .
$$

Now we can state and prove our main result of this section.

THEOREM 5.2. The variety $F$ satisfies the following.

(i) F is a cohomologically Tate variety.

(ii) $F$ has no polynomial count.

Proof. The proof of the first claim was given already in Example 4.3. Moreover, it follows from the description of the cohomology given there that the corresponding Hodge-Deligne polynomial is

$$
\begin{aligned}
H D_{F}(x, y)= & (x y)^{7}-9(x y)^{6}+36(x y)^{5}-82(x y)^{4} \\
& +119(x y)^{3}-110(x y)^{2}+60(x y)-15
\end{aligned}
$$

Assume that (ii) fails, that is, that $F$ has polynomial count with polynomial $P_{F}$. Then according to Katz's theorem (see [11]), we must have

$$
P_{F}(t)=t^{7}-9 t^{6}+36 t^{5}-82 t^{4}+119 t^{3}-110 t^{2}+60 t-15 .
$$

We will reach a contradiction by showing that $P_{F}(p) \neq A(p)$ for infinitely many primes, namely, for all the primes $p$ in the progression $12 b+11$ (Dirichlet prime number theorem). Let $p$ be such a prime, and write $p=4 k+3$ as above. Then a simple computation (e.g., using Maple) shows that

$$
P_{F}(4 k+3)=8(2 k+1)\left(1024 k^{6}+2560 k^{5}+2752 k^{4}\right.
$$

$$
\left.+1632 k^{3}+584 k^{2}+129 k+15\right) .
$$

Hence, to complete the proof, it is enough to show that $A(p) \not \equiv 0 \bmod 8$ for any prime $p$ as above. Using (5.13), this is equivalent to showing that

$$
2+n_{1}^{\prime} n_{2}^{\prime}-3 n_{1}^{\prime}-3 n_{2}^{\prime} \not \equiv 0 \bmod 4 \text {. }
$$

This in turn follows from the following. 
Lemma 5.3. Let $p$ be a prime number of the form $4 k+3$. Then both $n_{1}^{\prime}$ and $n_{2}^{\prime}$ are divisible by 4 .

Proof. Consider the group $G=\{ \pm 1\} \times\{1, \tau\}$ acting on the (finite) Milnor fiber $F_{1}(1)$ by

$$
\begin{aligned}
-1 \cdot\left(x_{1}, x_{2}, x_{3}\right) & =\left(-x_{1},-x_{2},-x_{3}\right), \\
\tau \cdot\left(x_{1}, x_{2}, x_{3}\right) & =\left(x_{2}, x_{1}, x_{3}\right),
\end{aligned}
$$

and

$$
-\tau \cdot\left(x_{1}, x_{2}, x_{3}\right)=\left(-x_{2},-x_{1},-x_{3}\right) .
$$

There are two types of $G$-orbits. First, we have the orbits $G x$, corresponding to points $x$ such that $x_{1} \neq x_{2}$. Then the isotropy group $G_{x}$ is trivial, and the orbit $G x$ consists of four points. Since we are interested in a computation modulo 4, we can forget about such orbits.

The second type of orbit corresponds to points $x$ such that $x_{1}=x_{2}=t$. Then $x \in F_{1}(1)$ is equivalent to

$$
\left(x_{3}+t\right)^{2}=\Delta(t)
$$

where $\Delta(t)=t^{2}+t^{-2}$. There are two possibilities.

(a) $\Delta(t) \notin H$; that is, $\Delta(t)$ is not a square in $\mathbb{F}_{p}^{*}$. Then (5.18) is impossible, and we do not get a point in $F_{1}(1)$.

(b) $\Delta(t)$ is a square in $\mathbb{F}_{p}^{*}$. Then (5.18) has two solutions, namely, $x_{3}=$ $-t+y$ and $x_{3}=-t-y$, with $y$ satisfying $y^{2}=\Delta(t)$. In this way we get two points in $F_{1}(1)$. Note that $\Delta(t)=\Delta(-t)$; hence, for the point $(-t)$ we are exactly in the same situation as for the point $t$. It follows that for each pair $\{t,-t\}$ (i.e., orbit of the obvious $\{ \pm 1\}$-action on $\mathbb{F}_{p}^{*}$ ), we get either 0 points in the (finite) Milnor fiber $F_{1}(1)$, or we get four points. This proves the claim for $n_{1}^{\prime}$.

Consider next the group $G=\{ \pm 1\} \times\{1, \tau\} \times\{1, \sigma\}$ acting on the (finite) Milnor fiber $F_{2}(1)$ by

$$
\begin{aligned}
-1 \cdot\left(x_{4}, x_{5}, x_{6}, x_{7}, x_{8}\right) & =\left(-x_{4},-x_{5},-x_{6},-x_{7},-x_{8}\right), \\
\tau \cdot\left(x_{4}, x_{5}, x_{6}, x_{7}, x_{8}\right) & =\left(x_{5}, x_{4}, x_{6}, x_{7}, x_{8}\right),
\end{aligned}
$$

and

$$
\sigma \cdot\left(x_{4}, x_{5}, x_{6}, x_{7}, x_{8}\right)=\left(x_{4}, x_{5}, x_{7}, x_{6}, x_{8}\right)
$$


(and their obvious consequences). Since we are interested in a computation modulo 4, we can forget about all orbits corresponding to points having an isotropy group of order at most 2 . Now let $x$ be a point such that $G_{x}=$ $\{1, \tau\} \times\{1, \sigma\}$. Then $x=\left(s, s, t, t, x_{8}\right)$ and $x \in F_{2}(1)$ is equivalent to

$$
\left(x_{8}+s+t\right)^{2}=\Delta(s, t),
$$

where $\Delta(t)=(s+t)^{2}+(s t)^{-2}$. Note that $\Delta(s, t)=\Delta(-s,-t)$; hence, for the point $(-s,-t)$ we are exactly in the same situation as for the point $(s, t)$. It follows that for each orbit of the obvious $\{ \pm 1\}$-action on $\left(\mathbb{F}_{p}^{*}\right)^{2}$, we get either zero points in the (finite) Milnor fiber $F_{2}(1)$, or we get four points. This proves the claim for $n_{2}^{\prime}$.

REMARK 5.4. Surprisingly, it seems that $P_{F}(p)=A(p)$ for many primes of the form $p=4 k+1$. Indeed, by a computer-aided computation, we have obtained the equality $P_{F}(p)=A(p)$ for the first 18 prime numbers of the form $p=4 k+1$.

REMARK 5.5. Kisin and Lehrer [13] have considered a notion of mixed Tate variety (imposing conditions on the eigenvalues of Frobenius action on $p$-adic étale cohomology) (see [13, Definition (2.6)]) and shown that if a variety $Y$ is mixed Tate, then $Y$ is cohomologically Tate (see [13, Theorem $(2.2)(2)])$. Then in [13, Remark 2.4, p. 213], they discuss the conjectural equivalence between cohomologically Tate and mixed Tate conditions. Any hyperplane arrangement complement is mixed Tate (see [13, Proposition 3.1.1]).

We say that $Y$ has weak polynomial count with count polynomial $P_{Y}$ if there is a polynomial $P_{Y} \in \mathbb{Z}[t]$ such that for all but finitely many primes $p$, there is an integer $k_{p}>0$ satisfying the following: if we set $q=p^{k_{p}}$, then for any finite field $\mathbb{F}_{q^{s}}$ with $s \in \mathbb{N}^{*}$, the number of points of $Y$ over $\mathbb{F}_{q^{s}}$ is precisely $P_{Y}\left(q^{s}\right)$.

Kisin and Lehrer have shown that if a variety $Y$ is mixed Tate, then $Y$ has weak polynomial count (see [13, Proposition 3.4]).

Acknowledgments. We would like to thank Nero Budur, Denis Ibadula, Mark Kisin, Gus Lehrer, and Morihiko Saito for useful discussions in relation to this work. Special thanks are also due to Gabriel Sticlaru, who provided expert help in some numerical experiments with rather large numbers (see Remark 5.4). 


\section{REFERENCES}

[1] N. Budur and M. Saito, Jumping coefficients and spectrum of a hyperplane arrangement, Math. Ann. 347 (2010), 545-579.

[2] D. C. Cohen, G. Denham, and A. I. Suciu, Torsion in Milnor fiber homology, Algebr. Geom. Topol. 3 (2003), 511-535.

[3] D. C. Cohen and A. I. Suciu, On Milnor fibrations of arrangements, J. Lond. Math. Soc. (2) 51 (1995), 105-119.

[4] P. Deligne, P. Griffiths, J. Morgan, and D. Sullivan, Real homotopy theory of Kähler manifolds, Invent. Math. 29 (1975), 245-274.

[5] A. Dimca, Singularities and Topology of Hypersurfaces, Springer, New York, 1992.

[6] - Sheaves in Topology, Springer, New York, 2004.

[7] A. Dimca and G. I. Lehrer, "Purity and equivariant weight polynomials" in Algebraic Groups and Lie Groups, Austral. Math. Soc. Lect. Ser. 9, Cambridge University Press, Cambridge, 1997, 161-181.

[8] - Hodge-Deligne equivariant polynomials and monodromy of hyperplane arrangements, preprint, arXiv:1006.3462 [math.AG]

[9] A. Dimca and S. Papadima, Finite Galois covers, cohomology jump loci, formality properties, and multinets, Ann. Sc. Norm. Super. Pisa Cl. Sci (5) 10 (2011), 253-268.

[10] A. Dimca, S. Papadima, and A. I. Suciu, Quasi-Kähler groups, 3-manifold groups, and formality, Math. Z. 268 (2011), 169-186.

[11] T. Hausel and F. Rodriguez-Villegas, Mixed Hodge polynomials of character varieties, with an appendix by Nicholas M. Katz, Invent. Math. 174 (2008), 555-624.

[12] M. Kim, Weights in cohomology groups arising from hyperplane arrangements, Proc. Amer. Math. Soc. 120 (1994), 697-703.

[13] M. Kisin and G. I. Lehrer, Eigenvalues of Frobenius and Hodge numbers, Pure Appl. Math. Q. 2 (2006), 497-518.

[14] G. I. Lehrer, The $\ell$-adic cohomology of hyperplane complements, Bull. Lond. Math. Soc. 24 (1992), 76-82.

[15] A. Libgober, "Eigenvalues for the monodromy of the Milnor fibers of arrangements" in Trends in Singularities, Trends Math., Birkhäuser, Basel, 2002, 141-150.

[16] - On combinatorial invariance of the cohomology of Milnor fiber of arrangements and Catalan equation over function field, preprint, arXiv:1011.0191 [math.AG]

[17] A. Némethi, Generalized local and global Sebastiani-Thom type theorems, Compos. Math. 80 (1991), 1-14.

[18] M. Oka, On the homotopy types of hypersurfaces defined by weighted homogeneous polynomials, Topology 12 (1973), 19-32.

[19] P. Orlik and R. Randell, The Milnor fiber of a generic arrangement, Ark. Mat. 31 (1993), 71-81.

[20] P. Orlik and L. Solomon, Combinatorics and topology of complements of hyperplanes, Invent. Math. 56 (1980), 167-189.

[21] P. Orlik and H. Terao, Arrangements of Hyperplanes, Grundlehren Math. Wiss. 30, Springer, Berlin, 1992.

[22] M. Saito, Multiplier ideals, b-functions, and spectrum of a hypersurface singularity, Compos. Math. 143 (2007), 1050-1068.

[23] K. Sakamoto, "Milnor fiberings and their characteristic maps" in Manifolds-Tokyo 1973 (Tokyo, 1973), Univ. Tokyo Press, Tokyo, 1975, 145-150. 
[24] V. Schechtman, H. Terao, and A. Varchenko, Local systems over complements of hyperplanes and the Kac-Kazhdan condition for singular vectors, J. Pure Appl. Algebra 100 (1995), 93-102.

[25] B. Z. Shapiro, The mixed Hodge structure of the complement to an arbitrary arrangement of affine complex hyperplanes is pure, Proc. Amer. Math. Soc. 117 (1993), 931-933.

[26] R. P. Stanley, "An introduction to hyperplane arrangements" in Geometric Combinatorics, IAS/Park City Math. Ser. 13, Amer. Math. Soc., Providence, 2007, 389-494.

[27] J. Steenbrink, Intersection form for quasi-homogeneous singularities, Compos. Math. 34 (1977), 211-223.

[28] D. Sullivan, Infinitesimal computations in topology, Publ. Math. Inst. Hautes Études Sci. 47 (1977), 269-331.

[29] D. Tapp, Picard-Lefschetz monodromy of products, J. Pure Appl. Algebra 212 (2008), 2314-2319.

[30] H. Zuber, Non-formality of Milnor fibres of line arrangements, Bull. Lond. Math. Soc. 42 (2010), 905-911.

Institut Universitaire de France et Laboratoire J.A. Dieudonné

UMR CNRS 7351

Université de Nice Sophia Antipolis

06108 Nice Cedex 02

France

dimca@unice.fr 